\title{
Desempenho de frangos de corte alimentados com diferentes níveis de suplementação de vitamina $E$
}

\author{
[Effect of vitamin E supplementation on the performance of broiler chickens]
}

M.A. Pompeu ${ }^{1}$, N.C. Baião $o^{2,5}$, L.J.C. Lara ${ }^{2}$, J.S.R. Rocha ${ }^{1}$, P.C. Cardeal ${ }^{1}$, R.C. Baião ${ }^{1}$, L.F.P. Pereira ${ }^{1}$, M.P.F. Teixeira ${ }^{1}$, V.M. Barbosa ${ }^{3}$, C.E. Cunha ${ }^{4}$

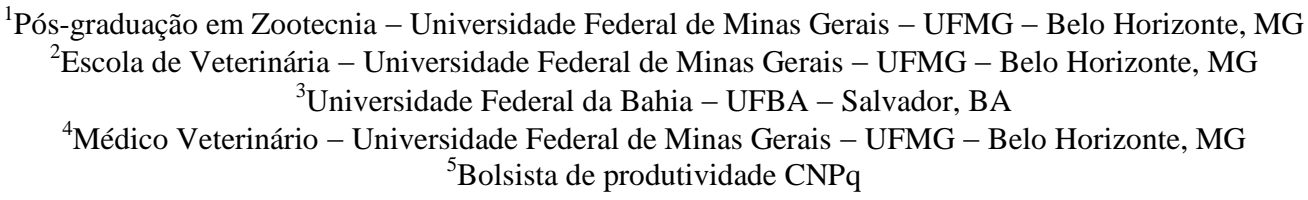

\section{RESUMO}

Objetivou-se a avaliação dos efeitos dos níveis de suplementação de vitamina E na ração para frangos de corte sobre o desempenho na fase inicial (um a 21 dias de idade) e de crescimento ( 21 a 39 dias de idade). Os tratamentos foram definidos pelos níveis de suplementação de vitamina E (DL- $\alpha$ tocoferol). As quantidades adicionadas às rações foram: 10; 30; 50; 75 e 100mg de vitamina $\mathrm{E}$ para cada $\mathrm{kg}$ de ração. $\mathrm{Na}$ fase inicial (experimento I), o menor nível de suplementação de vitamina E mostrou-se superior aos demais, apresentando efeito significativo $(\mathrm{P} \leq 0,05)$ sobre o peso médio aos 21 dias de idade e ganho de peso de um a 21 dias de idade. Na fase de crescimento (experimento II), não houve efeito significativo $(\mathrm{P}>0,05)$ dos níveis de suplementação de vitamina $\mathrm{E}$ sobre as variáveis estudadas. O nível mínimo de suplementação de vitamina $\mathrm{E}$ testado $(10 \mathrm{mg} / \mathrm{kg})$ é suficiente para atender às exigências dos frangos de corte machos sobre o desempenho.

Palavras-chave: aves, dieta, ganho de peso, tocoferol

\begin{abstract}
This study aimed to evaluate the effect of vitamin E supplementation on the performance of broiler chickens in the initial and growth phase, from one to 21 and 21 to 39 days of age, respectively. Five increasing levels of DL- $\alpha$ tocopherol supplementation (10,30,50, 75 and 100mg to each $\mathrm{kg}$ of diet) were added to the diet. In the initial phase (experiment I), the lowest vitamin E level was superior by presenting significant effect $(P \leq 0.05)$ on body weight at 21 days of age and weight gain from one to 21 days old. In the growth phase (experiment II), there was no significant effect $(P>0.05)$ of vitamin $E$ supplementation on the variables studied. The minimum level of vitamin E supplementation tested is sufficient to attend the requirements of male broiler chickens.
\end{abstract}

Keywords: bird, diet, tocopherol, weight gain

\section{INTRODUÇÃO}

De forma geral, as vitaminas são compostos químicos orgânicos que usualmente não são sintetizados pelas células animais, mas são necessárias para a mantença, o crescimento e a produção. Há 13 vitaminas frequentemente listadas como necessárias para as aves de

Recebido em 8 de maio de 2013

Aceito em 30 de julho de 2014

E-mail: marianapompeu@ hotmail.com produção, as quais ocorrem nos alimentos em quantidades variáveis e em diferentes combinações. Nem todos os alimentos contêm todas essas vitaminas, e alguns possuem maior quantidade de certas vitaminas do que de outras. Como as vitaminas são compostos químicos definidos, as produzidas comercialmente são tão valiosas quanto as encontradas em alimentos naturais (North e Bell, 1990). 
A vitamina E é essencial para a integridade reprodutiva, muscular, circulatória, nervosa e imunológica dos animais. É um importante antioxidante biológico, como também, o primeiro identificado (início do século XX). Nos tecidos atua em algumas funções básicas do mecanismo celular, ou com papel protetor intracelular antioxidante, prevenindo a oxidação das gorduras insaturadas e de outras substâncias sensíveis ao oxigênio, como a vitamina A e o ácido ascórbico. Age também como elemento importante no transporte direto de elétrons, ou indiretamente como possível agente parcial de ligação do citocromo redutase na cadeia respiratória. Para os galináceos, principalmente aves em crescimento e desenvolvimento do sistema nervoso central, a vitamina $\mathrm{E}$ possui grande importância no desempenho produtivo (Washington, 1960; Leshchinsky e Klasing, 2001).

O $\alpha$-tocoferol é, nutricionalmente, o representante mais importante do grupo de compostos com atividade de vitamina E, por apresentar maior atividade biológica, maior índice de absorção intestinal, maior deposição nos tecidos, menor excreção fecal e oxidação mais lenta quando comparado às demais formas encontradas (Rice e Kennedy, 1998).

Objetivou-se a avaliação dos efeitos dos níveis de suplementação de vitamina $E$ na ração para frangos de corte sobre o desempenho das aves na fase inicial (de um a 21 dias de idade) e de crescimento (de 21 a 39 dias de idade).

\section{MATERIAL E MÉTODOS}

Para a determinação do nível de suplementação da vitamina $\mathrm{E}$, foram realizados dois experimentos: o experimento I, para determinar o nível de suplementação na fase inicial (um a 21 dias de idade), e o experimento II, para avaliar o nível de suplementação na fase de crescimento (22 a 39 dias de idade). Os pintos para os dois experimentos foram alojados em galpão convencional, dividido em 60 boxes idênticos, sendo 30 boxes de cada lado.

Foram utilizados 900 pintos de um dia de idade, machos, da linhagem Cobb, para cada experimento. Para os experimentos, as aves foram divididas em cinco tratamentos com seis repetições de 30 aves. Porém, até as aves do experimento II atingirem a idade experimental (21 dias de idade), todas receberam a mesma ração à qual foi adicionada a quantidade utilizada comercialmente $(30 \mathrm{mg} / \mathrm{kg}$ de vitamina E) e, então, no $21^{\circ}$ dia, foram divididas em cinco tratamentos e seis repetições de 30 aves cada.

Utilizaram-se dois tipos de ração, de acordo com a fase de criação; o primeiro para a fase inicial e o segundo para a fase de crescimento (de um a 21 dias e de 22 a 39 dias de idade, respectivamente). As rações foram isonutritivas, com exceção dos níveis de vitamina E. Para a formulação das dietas, foram considerados os valores dos ingredientes estabelecidos por Rostagno et al. (2011) e os níveis nutricionais foram calculados de acordo com Lara et al. (2008). A composição das rações e seus níveis nutricionais encontram-se na Tab. 1.

Os tratamentos foram definidos pelos níveis de suplementação de vitamina E. As quantidades de vitamina $\mathrm{E}$ adicionadas às rações, tanto no experimento I quanto no experimento II, foram as seguintes: 10; 30; 50; 75 e 100mg de vitamina E para cada kg de ração.

As variáveis estudadas nos dois experimentos foram: peso corporal, consumo de ração, ganho de peso, conversão alimentar e taxa de viabilidade. Para as avaliações do desempenho, o delineamento experimental empregado foi $o$ inteiramente ao acaso, constituído por cinco tratamentos com seis repetições de 30 aves cada, com exceção da taxa de viabilidade, que violou os princípios da normalidade e da homocedasticidade e, dessa forma, o teste utilizado foi o Kruskal-Wallis. Os dados foram submetidos à análise de variância, para verificação dos efeitos significativos entre os fatores simples. O nível ideal de vitamina $\mathrm{E}$ foi obtido regredindo-se as variáveis respostas em relação aos níveis utilizados em seus componentes lineares e quadráticos, para a escolha do modelo de regressão que melhor descrevesse as observações (Sampaio, 2002). 
Tabela 1. Composição percentual e níveis nutricionais calculados das rações iniciais e de crescimento

\begin{tabular}{lcc}
\hline Ingredientes & Inicial & Crescimento \\
\hline Milho grão & 59,80 & 65,00 \\
Farelo de soja 46\% & 31,00 & 25,70 \\
Farinha de carne 42\% & 7,35 & 6,00 \\
Óleo de soja & 0,80 & 2,20 \\
Inerte & 0,19 & 0,14 \\
Sal comum & 0,30 & 0,30 \\
L-Lisina HCL & 0,155 & 0,21 \\
DL-Metionina & 0,175 & 0,170 \\
Suplemento vitamínico (sem vitamina E*) & 0,101 & $0,10^{2}$ \\
Cloreto de colina 60\% & 0,08 & 0,06 \\
Suplemento mineral ${ }^{3}$ & 0,05 & 0,05 \\
\hline Total (\%) & 100,00 & 100,00 \\
\hline & & 19,80 \\
Proteína bruta (\%) & Níveis Nutricionais & 3.090 \\
Energia metabolizável (kcal/kg) & 22,30 & 2,79 \\
Fibra bruta (\%) & 2.930 & 0,44 \\
Metionina digestível (\%) & 3,00 & 1,05 \\
Lisina digestível (\%) & 0,47 & 0,70 \\
Metionina + cistina digestível (\%) & 1,15 & 0,63 \\
Treonina digestível (\%) & 0,76 & 0,85 \\
Cálcio (\%) & 0,72 & 0,42 \\
Fósforo disponível (\%) & 0,99 & 0,19 \\
Sódio (\%) & 0,50 & 0,20 \\
\hline Vitama & & \\
\hline
\end{tabular}

*Vitamina $\mathrm{E}(\mathrm{dl}-\alpha$ tocoferil acetato $50 \%)=$ Tratamentos.

1 - Suplemento vitamínico inicial (concentração/kg de produto): vit. A: $9.000 \mathrm{UI}$; vit. D3: $2.500 .000 \mathrm{UI}$; vit. K3: $2.500 \mathrm{mg}$; vit. B1: $2.000 \mathrm{mg}$; vit. B2: $5.000 \mathrm{mg}$; vit. B6: 2.500mg; vit. B12: $14.000 \mathrm{mcg}$; biotina: 80mg; niacina: $35.000 \mathrm{mg}$; ácido fólico: $1.000 \mathrm{mg}$; ácido pantotênico: $12.000 \mathrm{mg}$.

2 - Suplemento vitamínico crescimento (concentração/kg de produto): vit. A: 9.000 UI; vit. D3: 500.000 UI; vit. K3: 500mg; vit. B1: 500mg; vit. B2: $1.000 \mathrm{mg}$; vit. B6: $1.000 \mathrm{mg}$; vit. B12: $5.000 \mathrm{mcg}$; biotina: $15 \mathrm{mg}$; niacina: $7.500 \mathrm{mg}$; ácido fólico: $250 \mathrm{mg}$; ácido pantotênico: $2.500 \mathrm{mg}$.

3 - Suplemento mineral inicial/crescimento (concentração/kg de produto): Mn: 90.000mg; Zn: 80.000mg; Fe: 30.000mg; Cu: 10.000mg; Se: 290mg.

O modelo de regressão empregado para a estimativa dos níveis de suplementação de vitamina $\mathrm{E}$ foi o linear: $\mathrm{Y}=\mathrm{a}+\mathrm{b} \mathrm{X}$; onde $\mathrm{Y}=$ variável estudada; $\mathrm{a}=$ coeficiente linear (corresponde ao ponto de interseção que a reta apresenta com o eixo vertical $\mathrm{Y}) ; \mathrm{b}=$ coeficiente de regressão ou angular (define a inclinação da reta), e $\mathrm{X}=$ nível de suplementação de vitamina E.

\section{RESULTADOS E DISCUSSÃO}

Os resultados do experimento $\mathrm{I}$, relativos ao desempenho da fase inicial dos frangos de corte, estão expressos na Tab. 2.
Os níveis de suplementação de vitamina E adotados não influenciaram as variáveis: peso aos sete dias (P7), consumo de ração (CR), conversão alimentar (CA) e viabilidade (VIA) dos frangos de corte na fase inicial de criação $(\mathrm{P}>0,05)$. No entanto, houve efeito significativo $(\mathrm{P} \leq 0,05)$ sobre o peso aos 21 dias $(\mathrm{P} 21)$ e o ganho de peso de um a 21 dias de idade (GP), que apresentaram efeito linear significativo, de acordo com as equações de regressão:

$\mathrm{P} 21=925,085-0,266 \times\left(\mathrm{R}_{2}\right.$ ajustado $\left.=70,14 \%\right)$ $\mathrm{GP}=886,289-0,263 \times\left(\mathrm{R}_{2}\right.$ ajustado $\left.=70,85 \%\right)$ 
Tabela 2. Peso inicial (PI), peso aos sete dias (P7), peso aos 21 dias (P21), consumo de ração (CR), ganho de peso (GP), conversão alimentar (CA) e viabilidade (VIA) dos frangos de corte na fase inicial de criação, de acordo com os níveis de suplementação de vitamina $\mathrm{E}$

\begin{tabular}{cccccccc}
\hline Vit. E $(\mathrm{mg} / \mathrm{kg})$ & $\mathrm{PI}(\mathrm{g})$ & $\mathrm{P} 7(\mathrm{~g})$ & $\mathrm{P} 21(\mathrm{~g})$ & $\mathrm{CR}(\mathrm{g})$ & GP $(\mathrm{g})$ & CA $(\mathrm{g} / \mathrm{g})$ & VIA $(\%)$ \\
\hline 10 & 38,75 & 178,33 & 925,59 & 1128,75 & 886,84 & 1,270 & 98,33 \\
30 & 38,62 & 177,39 & 914,39 & 1148,37 & 875,76 & 1,312 & 98,89 \\
50 & 38,88 & 170,11 & 913,62 & 1134,97 & 874,74 & 1,298 & 98,89 \\
75 & 38,31 & 174,62 & 897,64 & 1138,07 & 859,33 & 1,326 & 98,33 \\
100 & 38,55 & 174,86 & 903,67 & 1128,77 & 865,11 & 1,306 & 98,89 \\
\hline Regressão & $\mathrm{ns}$ & $\mathrm{ns}$ & $\mathrm{L}$ & $\mathrm{ns}$ & $\mathrm{L}$ & $\mathrm{ns}$ & $*$ \\
\hline CV $(\%)$ & 0,97 & 3,50 & 3,56 & 3,07 & 3,72 & 3,02 & - \\
\hline
\end{tabular}

ns $=$ Efeito não significativo pelo teste $\mathrm{F}(\mathrm{P}>0,05)$

$\mathrm{L}=$ Efeito significativo do modelo linear pelo teste $\mathrm{F}(\mathrm{P} \leq 0,05)$

*Efeito não significativo pelo teste de Kruskal-Wallis $(\mathrm{P}>0,05)$

Tanto o peso aos 21 dias (P21) quanto o ganho de peso de um a 21 dias de idade (GP) apresentaram a mesma tendência; com o aumento dos níveis de suplementação de vitamina $\mathrm{E}$, houve diminuição dos valores de peso aos 21 dias de idade e ganho de peso de um a 21 dias de idade, respectivamente. Dessa forma, o menor nível de suplementação de vitamina $\mathrm{E}$ avaliado $(10 \mathrm{mg} / \mathrm{kg})$ mostrou-se superior aos demais na fase inicial de criação.

Os resultados desta pesquisa se assemelham às descrições de Nobakht (2012). Este observou que a inclusão de $150 \mathrm{mg} / \mathrm{kg}$ de vitamina $\mathrm{E}$ na dieta para frangos de corte provocou efeitos negativos no desempenho, com redução no ganho de peso e consumo de ração, podendo ser justificado pelas interações (antagonismos) entre a vitamina A e E. Resultados contrários a estes foram relatados por Frigg (1990), Blum et al. (1992) e Barreto et al. (1999), que observaram melhora no desempenho dos frangos de corte, machos, com o aumento dos níveis de suplementação de vitamina E na ração.

Os valores obtidos com o experimento II, referentes ao desempenho dos frangos de corte no período de 21 a 39 dias de idade, estão apresentados na Tab. 3.

Tabela 3. Peso inicial (PI21), peso final (P39), consumo de ração (CR), ganho de peso (GP), conversão alimentar (CA) e viabilidade (VIA) dos frangos de corte na fase de crescimento, de acordo com os níveis de suplementação de vitamina $\mathrm{E}$

\begin{tabular}{ccccccc}
\hline Vit.E $(\mathrm{mg} / \mathrm{kg})$ & PI21 kg) & P39 kg) & CR $(\mathrm{kg})$ & GP $(\mathrm{kg})$ & $\mathrm{CA}(\mathrm{kg} / \mathrm{kg})$ & VIA $(\%)$ \\
\hline 10 & 0,910 & 2,83 & 3,04 & 1,92 & 1,586 & 99,44 \\
30 & 0,910 & 2,83 & 3,10 & 1,92 & 1,615 & 99,44 \\
50 & 0,909 & 2,83 & 2,98 & 1,92 & 1,547 & 98,88 \\
75 & 0,909 & 2,89 & 3,10 & 1,98 & 1,568 & 99,44 \\
100 & 0,907 & 2,80 & 3,00 & 1,89 & 1,587 & 97,22 \\
\hline Regressão & $\mathrm{ns}$ & $\mathrm{ns}$ & $\mathrm{ns}$ & $\mathrm{ns}$ & $\mathrm{ns}$ & $*$ \\
\hline CV $(\%)$ & 2,57 & 2,29 & 2,12 & 3,32 & 2,70 & - \\
\hline
\end{tabular}

ns = Efeito não significativo pelo teste $\mathrm{F}(\mathrm{P}>0,05)$

*Efeito não significativo pelo teste de Kruskal-Wallis $5 \%$

Não houve efeito significativo dos níveis de suplementação de vitamina E sobre o desempenho dos frangos de corte na fase de crescimento. Portanto, o menor nível de suplementação adotado $(10 \mathrm{mg} / \mathrm{kg})$ parece ser suficiente para atender às exigências dos frangos de corte nessa fase.
De forma semelhante, Souza et al. (2006), avaliando a influência de níveis de suplementação de vitamina $\mathrm{E}$ sobre o desempenho de frangos de corte, não encontraram efeito nos quatro níveis de suplementação testados $(0,100,150$ e $200 \mathrm{mg} / \mathrm{kg}$ de vitamina E), no período de um a 49 dias de idade. Esses resultados estão de acordo com Guo et al. (2001) e com os relatos de Nan et al. 
(1997), o que indica que, apesar de a vitamina $\mathrm{E}$ ser considerada uma importante ferramenta no aumento da imunidade em aves, efeitos positivos não foram detectados em parâmetros de desempenho.

Leonel et al. (2007) estudaram o efeito da suplementação de vitamina $\mathrm{E}$ na ração por diferentes períodos sobre o desempenho de frangos de corte. Foram utilizados seis tratamentos: dieta basal $(25 \mathrm{mg} / \mathrm{kg}$ de vitamina $\mathrm{E})$ e dieta suplementada com vitamina $\mathrm{E}$ $(300 \mathrm{mg} / \mathrm{kg})$ de um a 15 , um a 30 , um a 45 , 14 a 45 e 30 a 45 dias de idade. Não foram observadas diferenças significativas no desempenho das aves nos períodos estudados.

\section{CONCLUSÕES}

Nas condições em que foi conduzida esta pesquisa, conclui-se que o menor nível de suplementação de vitamina E utilizado $(10 \mathrm{mg} / \mathrm{kg})$ atende às exigências de frangos de corte machos nas fases inicial e de crescimento.

\section{AGRADECIMENTOS}

À Fundação de Amparo à Pesquisa do Estado de Minas Gerais (FAPEMIG) pelo financiamento ao projeto APQ-02233/10 que deu origem ao presente artigo científico. Ao Conselho Nacional de Desenvolvimento Científico e Tecnológico (CNPq) pela provisão da bolsa de estudos ao primeiro autor.

\section{REFERÊNCIAS}

BARRETO, S.L.T.; FERREIRA, W.M.; MORAES, T. Efeito de níveis de vitamina $\mathrm{E}$ na dieta sobre o desempenho e concentração de $\alpha$-tocoferol na carne de frangos de corte. Arq. Bras. Med. Vet. Zootec., v.51, p.387-392, 1999.

BLUM, J.C. Effect of dietary vitamin E supplie in broilers, male and female growth rate, viability, imune response, fat content and meat flavour variations during storage. Arch. Geflugelkd., v.56, p.37-42, 1992.

FRIGG, M. Effects of dietary vitamin E supplies in broilers. Evaluation of parameters related to oxidative stability of broiler meat. Nutr. Abst., v.22, p.24-30, 1990.
GUO, Y.; TANG, Q.; YUAN, J.; JIANG, Z. Effects of supplementation with vitamin $\mathrm{E}$ on the performance and the tissue peroxidation of broiler chicks and the stability of thigh meat against oxidative deterioration. Anim. Feed Sci. Technol., v.89, p.165-173, 2001.

LARA, L.J.C.; BAIÃO, N.C.; ROCHA, J.S.R. et al. Influência da forma física da ração e da linhagem sobre o desempenho e rendimento de cortes de frangos de corte. Arq. Bras. Med. Vet. Zootec., v.60, p.960968, 2008.

LEONEL, F.R.; OBA, A.; PELICANO, E.R.L. et al. Performance, carcass yield, and qualitative characteristics of breast and leg muscles of broilers fed diets supplemented with vitamin e at different ages. Braz. J. Poultry Sci., v.9, p.91-97, 2007.

LESHCHINSKY, T.V.; KLASING, K.C. Relationship between the level of dietary vitamin $\mathrm{E}$ and the immune response of broiler chickens. Poultry Sci., v.80, p.1590-1599, 2001.

NAN, T.K.; LEE, A.H.; MIN, S.B. et al. Influence of dietary supplementation with linseed and vitamin $\mathrm{E}$ on fatty acid, $\alpha$-tocopherol and lipid peroxidation in muscles of broiler chicks. Anim. Feed Sci. Technol., v.66, p.149-158, 1997.

NOBAKHT, A. The effects of different levels of poultry fat with vitamin $\mathrm{E}$ on performance and carcass traits of broilers. Afr. J. Agricult. Res., v.7, p.14201424, 2012.

NORTH, M.O.; BELL, D.D. Vitamins, minerals, and trace ingredients. In: NORTH, M.O. \& BELL, D.D. Commercial Chicken Production Manual. 4.ed., cap.26, NY: Chapman \& Hall, 1990. p.587-604.

RICE, D.; KENNEDY, S. Vitamin E: function and effects of deficiency. Br. Vet. J., v.144, p.482-496, 1998.

ROSTAGNO, H.S.; ALBINO, L.F.T.; DONZELE, J.L. et al. Tabelas brasileiras para aves e suínos: composição de alimentos e exigências nutricionais. 3.ed. Viçosa: Universidade Federal de Viçosa, 2011. 252p.

SAMPAIO, I.B.M. Estatística aplicada à experimentação animal. Belo Horizonte: FEPMVZ, 2. ed., 2002. 265p.

SOUZA, M.G.; OLIVEIRA, R.F.M.; DONZELE, J.L. et al. Utilização das vitaminas $\mathrm{C}$ e $\mathrm{E}$ em rações para frangos de corte mantidos em ambientes de alta temperatura. R. Bras. Zootec., v.40, p.2192-2198, 2011.

WASHINGTON, F.D. Vitamins and Hormones. cap.18, NY: Academic Press, 1960. p.43-87. 